\title{
Outage Probability of Macrodiversity Reception in the Presence of Fading and Weibull Co- Channel Interference
}

\author{
Miloš PERIĆ, Branimir JAKŠIĆ, Danijela ALEKSIĆ, Dragan RANDJELOVIĆ, Mihajlo STEFANOVIĆ
}

\begin{abstract}
In this paper macrodiversity reception with macrodiversity selection combining (SC) receiver and two microdiversity SC receivers operating over Gamma shadowed Weibull multipath fading channel in the presence of co-channel interference subjected to Weibull short term fading is analysed. Closed form expression for cumulative distribution function (CDF) of signals at outputs of microdiversity receivers is calculated. These results are used for CDF evaluation of macrodiversity SC receiver output signal envelope. Series of derived expressions for outage probability rapidly convergence from 5 to 10 terms should be summed to achieve accuracy at $5^{\text {th }}$ significant digit for any Gamma long term fading parameters and Weibull short term fading parameters. In this paper, influence of Gamma long term fading severity parameter, Gamma long term fading correlation coefficient and Weibull short term fading nonlinearity parameter on outage probability are analysed and discussed.
\end{abstract}

Keywords: co-channel interference; cumulative distribution function (CDF); macrodiversity (MAD); microdiversity (MID); outage probability; Weibull

\section{INTRODUCTION}

Macrodiversity (MAD) technique with MAD selection combining (SC) receiver and two or more microdiversity (MID) receivers can be used to reduce long term fading effects and short term fading effects on system performance of wireless communication system. MAD SC receiver selects MID SC receiver with higher signal envelope average power at inputs to provide service to user resulting in long term fading reduction and MID SC receiver selects and outputs branch with the highest signal to interference envelopes ratio reduction of short term fading effects reduction [1 - 4].

There are multiple distributions that can be used to describe small scale envelope variations in multithreaded Weibull distribution faded channels describing the variation of small scale envelope variations in nonlinear and non-line-of-sight multistage channels with a single cluster. This distribution has parameter $\alpha$ which is in relation to nonlinearity of fading channels. When parameter $\alpha$ is two, Gamma shadowed Weibull fading channel becomes Gamma shadowed Rayleigh multipath fading channel $[5,6]$. Long scale signal envelope variation can be described using Log-normal distribution or Gamma distribution. When long term fading is described by using Gamma distribution, outage probability can be derived in closed form. Gamma distribution has severity fading parameter $c$. When parameter $c$ goes to infinity, Gamma shadowed multipath fading channel becomes multipath fading channel. When correlation coefficient goes to one, the least signal occurs simultaneously on both MID receivers resulting in system performance degradation $[7,8]$.

There are more works in open technical literature considering performance of wireless communication system in the presence of short term fading, long term fading and co-channel interference. In [9], average level crossing rate and average fade duration of MAD system with MAD SC receiver and two MID maximal ratio combining (MRC) receivers operating over Gamma shadowed Nakagami-m multipath fading channel are calculated. Wireless communication system with SC receiver operating over Nakagami-m multipath fading channel in the presence of Nakagami-m co-channel interference is considered. In this paper, probability density function, cumulative distribution function, moments, outage probability and bit error probability of considered wireless system are evaluated. In [10], the second order statistics of MAD system are analysed.

In this paper, MAD system with MAD SC receiver and two MID SC receivers is studied. Received signal propagates over correlated Gamma shadowed Weibull multipath fading channel and co-channel interference. Closed form expression for cumulative distribution function of MAD SC receiver output signal envelope is calculated. To the best author's knowledge, MAD system with two MID receptions operating over Gamma shadowed Weibull fading channel in the presence of Weibull co-channel interference is not reported in open technical literature.

\section{SYSTEM MODEL}

MAD technique with MAD SC receiver and two MID receivers operating over Gamma shadowed Weibull multipath fading channel in the presence of co-channel interference subjected to Weibull short term fading is considered. Model MAD system is shown in Fig. 1. Received signals envelopes and co-channel interference envelope at inputs and outputs of MAD and MID receiver are denoted in Fig. 1.

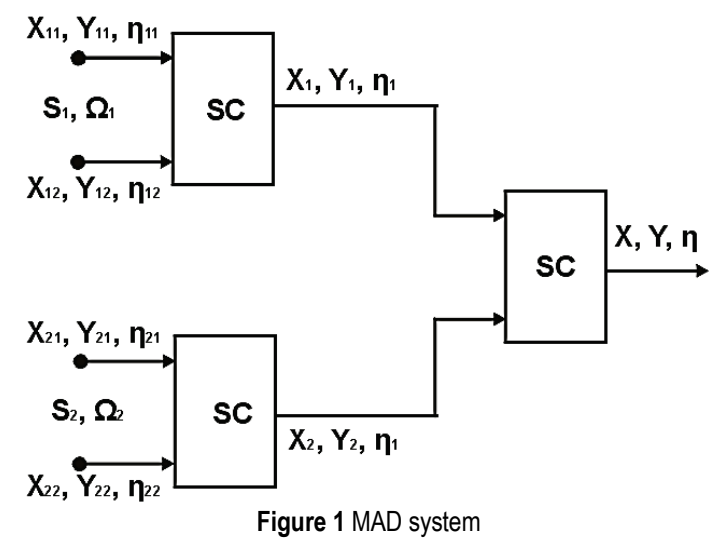

Received signal envelopes at inputs in the first MID receiver are denoted with $x_{11}$ and $x_{12}$ and the inputs in the second MID receiver with $x_{21}$ and $x_{22}$. The co-channel 
interference envelopes at inputs of the first MID SC receiver are denoted with $y_{11}$ and $y_{12}$, at inputs in the second MID receiver with $y_{21}$ and $y_{22}$. Ratios signal to interference are denoted with $\eta_{11}, \eta_{12}, \eta_{21}$ and $\eta_{22}$. At outputs of MID SC receivers desired signal envelopes are denoted with $x_{1}$ and $x_{2}$, co-channel interference envelope with $y_{1}$ and $y_{2}$ and ratios of signal to interference are denoted with $\eta_{1}$ and $\eta_{2}$. At output of MAD SC receiver desired signal is denoted with $x$, interference with $y$ and $\eta$ is signal to interference ratio. At inputs of MID SC receivers desired signal average power is denoted with $\Omega_{1}$ and $\Omega_{2}$ and interference signal average power with $S_{1}$ and $\mathrm{S}_{2}$.

Probability density function (PDF) of $x_{i j}, i=1,2 ; j=1,2$ is [3]:

$p_{x_{i j}}\left(x_{i j}\right)=\frac{\alpha}{\Omega_{i}} x_{i j}^{\alpha-1} \exp \left(-\frac{x_{i j}^{\alpha}}{\Omega_{i}}\right), i=1,2, j=1,2 ; \quad x_{i j} \geq 0 .(1)$

Probability density function (PDF) of $y_{i j}, i=1,2 ; j=1,2$ is

$p_{y_{i j}}\left(y_{i j}\right)=\frac{\alpha}{S_{i}} y_{i j}^{\alpha-1} \exp \left(-\frac{y_{i j}^{\alpha}}{S_{i}}\right), i=1,2, j=1,2 ; y_{i j} \geq 0$

The ratio of derived signal envelope end co-channel interference signal envelope is

$\eta_{i j}=\frac{x_{i j}}{y_{i j}}, x_{i j}=\eta_{i j} y_{i j}$

The PDF of $\eta_{i j}$ is

$p_{\eta_{i j}}\left(\eta_{i j}\right)=\int_{0}^{\infty} \mathrm{d} y_{i j} y_{i j} p_{y_{i j}}\left(y_{i j}\right) p_{x_{i j}}\left(x_{i j}\right)=$

$=\frac{\alpha^{2}}{S_{i} \Omega_{i}} \int_{0}^{\infty} \mathrm{d} y_{i j} y_{i j}^{2 \alpha-1} \exp \left(-y_{i j}^{\alpha}\left(\frac{\eta_{i j}}{\Omega_{l}}+\frac{1}{S_{i}}\right)\right)=$

$=\alpha\left(S_{i} \Omega_{i}\right)^{\alpha-1} \frac{\eta_{i j}^{\alpha-1}}{\left(\Omega_{i}+S_{i} \eta_{i j}^{\alpha}\right)^{\alpha}}$

The cumulative distribution function (CDF) of $\eta_{i j}$ is

$F_{\eta_{i j}}\left(\eta_{i j}\right)=\int_{0}^{\eta_{i j}} \mathrm{~d} t p_{\eta_{i j}}(t)=\alpha\left(S_{i} \Omega_{i}\right)^{\alpha-1} \int_{0}^{\eta_{i j}} \mathrm{~d} t \frac{t^{\alpha-1}}{\left(\Omega_{i}+S_{i} t^{\alpha}\right)^{\alpha}}$

After substituting $\Omega_{i}+S_{i} t^{\alpha}=z$ and $t^{\alpha-1} \mathrm{~d} t=\frac{1}{\alpha S_{i}} \mathrm{~d} z$ in (5), CDF of $\eta_{i j}$ becomes

$$
\begin{aligned}
& F_{\eta_{i j}}\left(\eta_{i j}\right)=\alpha\left(S_{i} \Omega_{i}\right)^{\alpha-1} \frac{1}{\alpha S_{i}} \int_{\Omega_{i}}^{\Omega_{i j}+S_{i} \eta_{i j}^{\alpha}} \frac{\mathrm{d} t}{t^{\alpha}}= \\
& =\Omega_{i}^{\alpha-1} S_{i}^{\alpha-2}\left(\frac{1}{\Omega_{i}^{\alpha-1}}-\frac{1}{\left(\Omega_{i}+S_{i} \eta_{i j}^{\alpha}\right)^{\alpha-1}}\right)= \\
& =S_{i}^{\alpha-2}\left(1-\frac{\Omega_{i}^{\alpha-1}}{\left(\Omega_{i}+S_{i} \eta_{i j}^{\alpha}\right)^{\alpha-1}}\right)
\end{aligned}
$$

The CDF of $\eta_{i}, i=1,2$ is

$$
F_{\eta_{i}}\left(\eta_{i}\right)=F_{\eta_{i 1}}\left(\eta_{i}\right) F_{\eta_{i 2}}\left(\eta_{i}\right)=S_{i}^{2 \alpha-2}\left(1-\frac{\Omega_{i}^{\alpha-1}}{\left(\Omega_{i}+S_{i} \eta_{i j}^{\alpha}\right)^{\alpha-1}}\right)
$$

Random variables $\Omega_{1}$ and $\Omega_{2}$ have correlated Gamma distribution

$$
\begin{aligned}
& p_{\Omega_{1} \Omega_{2}}\left(\Omega_{1} \Omega_{2}\right)=\frac{1}{\Gamma(c)\left(1-\rho^{2}\right) \rho^{\frac{c-1}{2}} \Omega_{0}^{c+1}} \times \\
& \times \sum_{i_{1}=0}^{\infty}\left(\frac{\rho}{\Omega_{0}\left(1-\rho^{2}\right)}\right)^{2 i_{1}+c-1} \frac{1}{i_{1} ! \Gamma\left(i_{1}+c\right)} \Omega_{1}^{i_{1}+c-1} \Omega_{2}^{i_{1}+c-1}
\end{aligned}
$$

where $c$ is Gamma long term fading severity parameter, $\rho$ is Gamma long term fading correlation coefficient and $\Omega$ is average value of $\Omega_{1}$ or $\Omega_{2}$. Random variable $S_{1}$ and $S_{2}$ follow also uncorrelated Gamma distribution

$$
\begin{aligned}
& p_{\mathrm{S}_{1} S_{2}}\left(S_{1} S_{2}\right)=\frac{1}{\Gamma\left(c_{1}\right) \beta^{c_{1}}} S_{1}^{c_{1}-1} \exp \left(-\frac{1}{\beta} S_{1}\right) \times \\
& \times \frac{1}{\Gamma\left(c_{1}\right) \beta^{c_{1}}} S_{2}^{c_{1}-1} \exp \left(-\frac{1}{\beta} S_{2}\right)
\end{aligned}
$$

where $c_{1}$ is Gamma parameter and $\beta$ is average value of $S_{1}$ or $S_{2}$.

\section{ANALYTICAL RESULTS}

MAD SC receiver selects MID SC receivers with higher signal envelope average power at inputs to provide service to user. Therefore, the CDF of $\eta$ is

$$
\begin{aligned}
& F_{\eta}\left(\frac{\eta}{S}\right)=\int_{0}^{\infty} \mathrm{d} \Omega_{1} \int_{0}^{\Omega_{1}} \mathrm{~d} \Omega_{2} F_{\eta_{1}}\left(\frac{\eta}{\Omega_{1}, S_{1}}\right) p_{\Omega_{1} \Omega_{2}}\left(\Omega_{1} \Omega_{2}\right)+ \\
& +\int_{0}^{\infty} \mathrm{d} \Omega_{2} \int_{0}^{\Omega_{2}} \mathrm{~d} \Omega_{1} F_{\eta_{2}}\left(\frac{\eta}{\Omega_{2}, S_{2}}\right) p_{\Omega_{1} \Omega_{2}}\left(\Omega_{1} \Omega_{2}\right)= \\
& =\int_{0}^{\infty} \mathrm{d} \Omega_{1} \int_{0}^{\Omega_{1}} \mathrm{~d} \Omega_{2} F_{\eta_{1}}\left(\frac{\eta}{\Omega_{1}, S_{1}}\right) p_{\Omega_{1} \Omega_{2}}\left(\Omega_{1} \Omega_{2}\right)=
\end{aligned}
$$




$$
\begin{aligned}
& =2 S_{i}^{2 \alpha-2} \frac{1}{\Gamma(c)\left(1-\rho^{2}\right) \rho^{\frac{c-1}{2}} \Omega_{0}^{c+1}} \times \\
& \times \sum_{i_{1}=0}^{\infty}\left(\frac{\rho}{\Omega_{0}\left(1-\rho^{2}\right)}\right)^{2 i_{1}+c-1} \frac{1}{i_{1} ! \Gamma\left(i_{1}+c\right)} \cdot \frac{1}{i_{1}+c} \times \\
& \times \sum_{j_{1}=0}^{\infty} \frac{1}{\left(i_{1}+c+1\right)_{\left(j_{1}\right)}} \cdot \frac{1}{\left(\Omega_{0}\left(1-\rho^{2}\right)\right)^{j}} \times\left(I_{1}-I_{2}+I_{3}\right)
\end{aligned}
$$

where $(a)_{n}$ is denoting the Pochhammer symbol [11].

The integral $I_{1}$ is

$$
\begin{aligned}
& I_{1}=\int_{0}^{\infty} \mathrm{d} \Omega_{1} \Omega_{1}^{2 i_{1}+2 c-1+j_{1}} \exp \left(-\frac{2 \Omega_{1}}{\Omega_{0}\left(1-\rho^{2}\right)}\right)= \\
& =\left(\frac{\Omega_{0}\left(1-\rho^{2}\right)}{2}\right)^{2 i_{1}+2 c+j_{1}} \Gamma\left(2 i_{1}+2 c+j_{1}\right)
\end{aligned}
$$

The integral $I_{2}$ is

$$
\begin{aligned}
& I_{2}=2 \int_{0}^{\infty} \mathrm{d} \Omega_{1} \Omega_{1}^{2 i_{1}+2 c-1+j_{1}+\alpha-1} \times \\
& \times \exp \left(-\frac{2 \Omega_{1}}{\Omega_{0}\left(1-\rho^{2}\right)}\right) \frac{1}{\left(\Omega_{1}+S_{1} \eta^{\alpha}\right)^{\alpha-1}}
\end{aligned}
$$

After substituting $\frac{\Omega_{1}}{S_{1} \eta^{\alpha}}=z, \quad \Omega_{1}=S_{1} \eta^{\alpha} z \quad$ and $\mathrm{d} \Omega_{1}=S_{1} \eta^{\alpha} \mathrm{d} z$, the integral $I_{2}$ becomes

$$
\begin{aligned}
& I_{2}=2\left(S_{1} \eta^{\alpha}\right)^{2 i_{1}+2 c+j_{1}+\alpha-1} \frac{1}{\left(S_{1} \eta^{\alpha}\right)^{\alpha-1}} \int_{0}^{\infty} \mathrm{d} z z^{2 i_{1}+2 c-1+j_{1}+\alpha-1} \times \\
& \times \exp \left(-\frac{2 S_{1} \eta^{\alpha}}{\Omega_{0}\left(1-\rho^{2}\right)} z\right) \frac{1}{(1+z)^{\alpha-1}}
\end{aligned}
$$

Previously integral can be solved by using formulas for confluent hypergeometric function $U(a, b, z)[11]$ :

$U(a, b, s)=\frac{1}{\Gamma(a)} \int_{0}^{\infty} \mathrm{d} t t^{\alpha-1} \exp (-s t) \frac{1}{(1+t)^{a+1-b}}$

For considered case, parameters $a, b$ and $s$ are

$$
\begin{aligned}
& a=2 i_{1}+2 c+j_{1}+\alpha-1 \\
& b=2 i_{1}+2 c+j_{1}+\alpha-1+1-\alpha+1=2 i_{1}+2 c+j_{1}+1 \\
& s=\frac{S_{1} \eta^{\alpha}}{\Omega_{0}\left(1-\rho^{2}\right)}
\end{aligned}
$$

After substituting Eq. (14), Eq. (15), Eq. (16) and Eq. (17) in Eq. (13), the expression for $I_{2}$ becomes

$$
\begin{aligned}
& I_{2}=2\left(S_{1} \eta^{\alpha}\right)^{2 i i^{2}+2 c+j 1} \Gamma\left(2 i_{1}+2 c+j_{1}+\alpha-1\right) \times \\
& \times U\left(2 i_{1}+2 c+j_{1}+\alpha-1,2 i_{1}+2 c+j_{1}+1, \frac{2 \eta^{\alpha}}{\Omega_{0}\left(1-\rho^{2}\right)} S_{1}\right)
\end{aligned}
$$

The integral $I_{3}$ is

$$
\begin{aligned}
& I_{3}=2\left(S_{1} \eta^{\alpha}\right)^{2 i_{1}+2 c+j_{1}} \Gamma\left(2 i_{1}+2 c+j_{1}+2 \alpha-2\right) \times \\
& \times U\left(2 i_{1}+2 c+j_{1}+2 \alpha-2,2 i_{1}+2 c+j_{1}+1, \frac{2 \eta^{\alpha}}{\Omega_{0}\left(1-\rho^{2}\right)} S_{1}\right)
\end{aligned}
$$

CDF of $\eta$ can be calculated by averaging CDF of $\eta / S_{1}$ rejected to $S_{1}$

$$
\begin{aligned}
& F_{\eta}(\eta)=\int_{0}^{\infty} \mathrm{d} S_{1} p_{S_{1}}\left(S_{1}\right) F_{\eta}\left(\frac{\eta}{S_{1}}\right)= \\
& =\frac{1}{\Gamma(c)\left(1-\rho^{2}\right) \rho^{\frac{c-1}{2}} \Omega_{0}^{c+1}} \sum_{i_{1}=0}^{\infty}\left(\frac{\rho}{\Omega_{0}\left(1-\rho^{2}\right)}\right)^{2 i_{1}+c-1} \times \\
& \times \frac{1}{i_{1} ! \Gamma\left(i_{1}+c\right)} \frac{1}{i_{1}+c} \sum_{j_{1}=0}^{\infty} \frac{1}{\left(i_{1}+c+1\right)_{\left(j_{1}\right)}} \times \\
& \times \frac{1}{\left(\Omega_{0}\left(1-\rho^{2}\right)\right)^{j_{1}}} \frac{1}{\Gamma\left(c_{1}\right) \beta^{c 1}} \times\left(I_{4}+I_{5}+I_{6}\right)
\end{aligned}
$$

The integral $I_{4}$ is

$$
\begin{aligned}
& I_{4}=\int_{0}^{\infty} \mathrm{d} S_{1} S_{1}^{c_{1}-1+\alpha-2} I_{1} \exp \left(-\frac{1}{\beta} S_{1}\right)= \\
& =\int_{0}^{\infty} \mathrm{d} S_{1} S_{1}^{c_{1}-1+\alpha-2} \exp \left(-\frac{1}{\beta} S_{1}\right) \times \\
& \times\left(\frac{\Omega_{0}\left(1-\rho^{2}\right)}{2}\right)^{2 i_{1}+2 c+j_{1}} \Gamma\left(2 i_{1}+2 c+j_{1}\right)
\end{aligned}
$$

The integral $I_{5}$ is

$$
\begin{aligned}
& I_{5}=\int_{0}^{\infty} d S_{1} S_{1}^{c_{1}-1+\alpha-2} I_{2} \exp \left(-\frac{1}{\beta} S_{1}\right)= \\
& =2 \eta_{1}^{4 i_{1}+4 c+2 j_{1}} \Gamma\left(2 i_{1}+2 c+j_{1}+\alpha-1\right) \times
\end{aligned}
$$$$
\times \int_{0}^{\infty} d S_{1} S_{1}^{c_{1}-1+2 \alpha-2+2 i_{1}+2 c+j 1} \exp \left(-\frac{1}{\beta} S_{1}\right) \times
$$

$\times U\left(2 i_{1}+2 c+j_{1}+\alpha-1,2 i_{1}+2 c+j_{1}+1, \frac{2 \eta^{\alpha}}{\Omega_{0}\left(1-\rho^{2}\right)} S_{1}\right)$

After substituting $\frac{2 \eta^{\alpha}}{\Omega_{0}\left(1-\rho^{2}\right)} S_{1}=z, S_{1}=\frac{\Omega_{0}\left(1-\rho^{2}\right)}{2 \eta^{\alpha}} z$ and $\mathrm{d} S_{1}=\frac{\Omega_{0}\left(1-\rho^{2}\right)}{2 \eta^{\alpha}} \mathrm{d} z$ in Eq. (22), the expression for $I_{5}$ becomes 


$$
\begin{aligned}
& I_{5}=2 \eta_{1}^{4 i_{1}+4 c+2 j_{1}} \Gamma\left(2 i_{1}+2 c+j_{1}+\alpha-1\right) \times \\
& \times\left(\frac{\Omega_{0}\left(1-\rho^{2}\right)}{2 \eta^{\alpha}}\right)^{c_{1}+2 \alpha-2+2 i_{1}+2 c+j_{1}} \\
& \times \\
& \times \int_{0}^{\infty} \mathrm{d} z z^{c_{1}-1+2 \alpha-2+2 i_{1}+2 c+j_{1}} \exp \left(-\frac{\Omega_{0}\left(1-\rho^{2}\right)}{2 \eta^{\alpha} \beta} z\right) \times \\
& \times U\left(2 i_{1}+2 c+j_{1}+\alpha-1,2 i_{1}+2 c+j_{1}+1, z\right)
\end{aligned}
$$

Previously integral can be solved by using formulas

$$
\begin{aligned}
& \int_{0}^{\infty} \mathrm{d} t t^{b-1} \exp (-s t) U(a, c, t)=\frac{\Gamma(b) \Gamma(b-c+1)}{\Gamma(a+b-c+1)} \times \\
& \times{ }_{2} F_{1}(b, b-c+1, a+b-c+1 ; 1-s)
\end{aligned}
$$

where ${ }_{2} F_{1}$ is hypergeometric function [11].

For considered case is

$$
\begin{aligned}
& b=c_{1}+2 \alpha-2+2 i_{1}+2 c+j_{1} \\
& b-c+1=c_{1}+2 \alpha-2+2 i_{1}+2 c+j_{1}-2 i_{1}- \\
& -2 c-j_{1}-1+1=c_{1}+2 \alpha-2 \\
& a+b-c+1=2 i_{1}+2 c+j_{1}-2 i_{1}+c_{1}+2 \alpha-2= \\
& =2 i_{1}+2 c+j_{1}+c_{1}+3 \alpha-3
\end{aligned}
$$

After substituting Eq. (24), Eq. (25), Eq. (26) and Eq. (27) in Eq. (23), the expression for $I_{5}$ becomes

$$
\begin{aligned}
& I_{5}=2 \eta_{1}^{4 i_{1}+4 c+2 j_{1}} \Gamma\left(2 i_{1}+2 c+j_{1}+\alpha-1\right) \times \\
& \times\left(\frac{\Omega_{0}\left(1-\rho^{2}\right)}{2 \eta^{\alpha}}\right)^{c_{1}+2 \alpha-2+2 i_{1}+2 c+j_{1}} \times \\
& \times \frac{\Gamma\left(c_{1}+2 \alpha-2+2 i_{1}+2 c+j_{1}\right) \Gamma\left(c_{1}+2 \alpha-2\right)}{\Gamma\left(2 i_{1}+2 c+j_{1}+c_{1}+3 \alpha-3\right)} \times \\
& \times{ }_{2} F_{1}\left(c_{1}+2 \alpha-2+2 i_{1}+2 c+j_{1}, c_{1}+2 \alpha-2,\right. \\
& \left.2 i_{1}+2 c+j_{1}+c_{1}+3 \alpha-3 ; 1-\frac{\Omega_{0}\left(1-\rho^{2}\right)}{2 \eta^{\alpha} \beta}\right)
\end{aligned}
$$

The integral $I_{6}$ is

$$
\begin{aligned}
& I_{6}=\int_{0}^{\infty} \mathrm{d} S_{1} S_{1}^{c_{1}-1+\alpha-2} I_{3} \exp \left(-\frac{1}{\beta} S_{1}\right)= \\
& =\eta_{1}^{4 i_{1}+4 c+2 j_{1}} \Gamma\left(2 i_{1}+2 c+j_{1}+\alpha-1\right) \times \\
& \times \int_{0}^{\infty} \mathrm{d} S_{1} S_{1}^{c_{1}-1+2 \alpha-2+2 i_{1}+2 c+j_{1}} \exp \left(-\frac{1}{\beta} S_{1}\right) \times \\
& \times U\left(2 i_{1}+2 c+j_{1}+2 \alpha-2,2 i_{1}+2 c+j_{1}+1, \frac{2 \eta^{\alpha}}{\Omega_{0}\left(1-\rho^{2}\right)} S_{1}\right)
\end{aligned}
$$

After substituting $\frac{2 \eta^{\alpha}}{\Omega_{0}\left(1-\rho^{2}\right)} S_{1}=z, S_{1}=\frac{\Omega_{0}\left(1-\rho^{2}\right)}{2 \eta^{\alpha}} z$ and $\mathrm{d} S_{1}=\frac{\Omega_{0}\left(1-\rho^{2}\right)}{2 \eta^{\alpha}} \mathrm{d} z$ in (22), the expression for $I_{6}$ becomes

$$
\begin{aligned}
& I_{6}=\eta_{1}^{4 i_{1}+4 c+2 j_{1}} \Gamma\left(2 i_{1}+2 c+j_{1}+\alpha-1\right) \times \\
& \times\left(\frac{\Omega_{0}\left(1-\rho^{2}\right)}{2 \eta^{\alpha}}\right)^{c_{1}+2 \alpha-2+2 i_{1}+2 c+j_{1}} \times \\
& \times \int_{0}^{\infty} \mathrm{d} z z^{c_{1}-1+2 \alpha-2+2 i_{1}+2 c+j_{1}} \exp \left(-\frac{\Omega_{0}\left(1-\rho^{2}\right)}{2 \eta^{\alpha} \beta} z\right) \times \\
& \times U\left(2 i_{1}+2 c+j_{1}+2 \alpha-2,2 i_{1}+2 c+j_{1}+1, z\right)= \\
& =\eta_{1}^{4 i_{1}+4 c+2 j_{1}} \Gamma\left(2 i_{1}+2 c+j_{1}+\alpha-1\right) \times \\
& \times\left(\frac{\Omega_{0}\left(1-\rho^{2}\right)}{2 \eta^{\alpha}}\right)^{c_{1}+2 \alpha-2+2 i_{1}+2 c+j_{1}} \times \\
& \times \frac{\Gamma\left(c_{1}+2 \alpha-2+2 i_{1}+2 c+j_{1}\right) \Gamma\left(c_{1}+2 \alpha-2\right)}{\Gamma\left(2 i_{1}+2 c+j_{1}+c_{1}+4 \alpha-4\right)} \times \\
& \times{ }_{2} F_{1}\left(c_{1}+2 \alpha-2+2 i_{1}+2 c+j_{1}, c_{1}+2 \alpha-2,\right. \\
& \left.2 i_{1}+2 c+j_{1}+c_{1}+4 \alpha-4 ; 1-\frac{\Omega_{0}\left(1-\rho^{2}\right)}{2 \eta^{\alpha} \beta}\right)
\end{aligned}
$$

\section{NUMERICAL RESULTS}

Cumulative distribution function (CDF) of considered NAD system in terms of signal to interference ratio (SIR) for different values of Gamma long term fading severity parameter $c$ and Gamma long term fading correlation coefficient $\rho$ is shown in Fig. 2.

CDF increases as SIR increases and outage probability goes to one for high values of SIR or when SIR goes to infinity. The influence of SIR on outage probability is higher for lower values of SIR. When severity parameter $c$ increases, outage probability decreases resulting in system performance improvement. The influence of parameter $c$ on outage probability is higher for lower values of SIR, also, the influence of severity parameter $c$ on outage probability is higher for lower values of parameter $c$. When correlation coefficient decreases, CDF decreases and performance quality is worse. The influence of correlation coefficient on outage probability is higher for lower values of SIR. The influence of correlation coefficient on outage probability is similar for lower and higher values of parameter $c$.

Cumulative distribution function versus Gamma long term fading severity parameter $c$ for different values of Gamma long term fading correlation coefficient $\rho$ is shown in Fig. 3. CDF decreases as parameter $\mathrm{c}$ increases and $\mathrm{CDF}$ fast decreases to lower values of correlation coefficients.

In the numerical analysis, standard values of the system parameters are used [3]. Values of Gamma long term fading severity parameter $c$ go from 1 to 3 . Values of Gamma long term fading correlation coefficient $\rho$ go from 0 to 1 . 


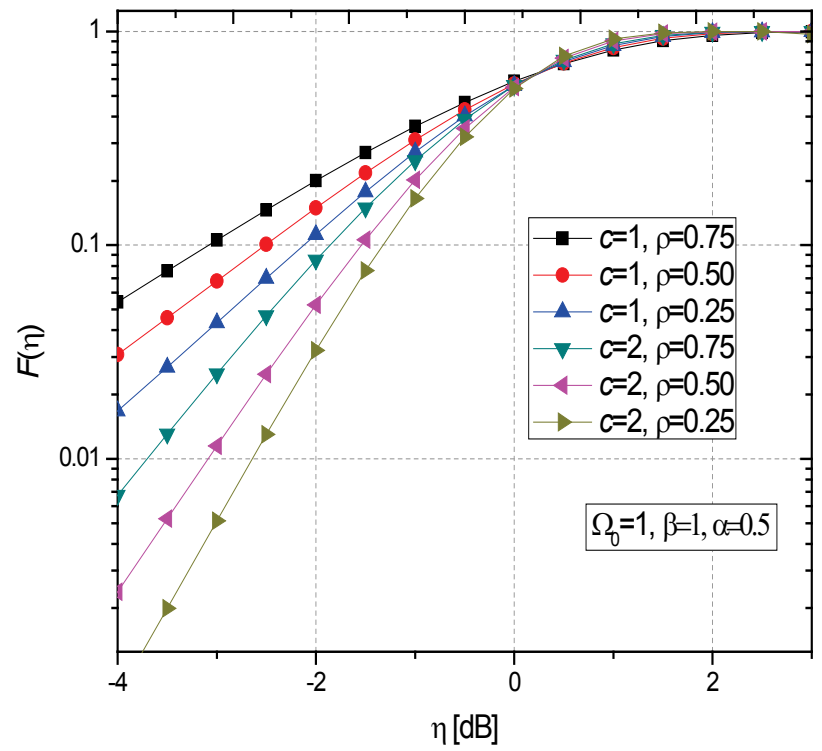

Figure 2 Cumulative distribution function

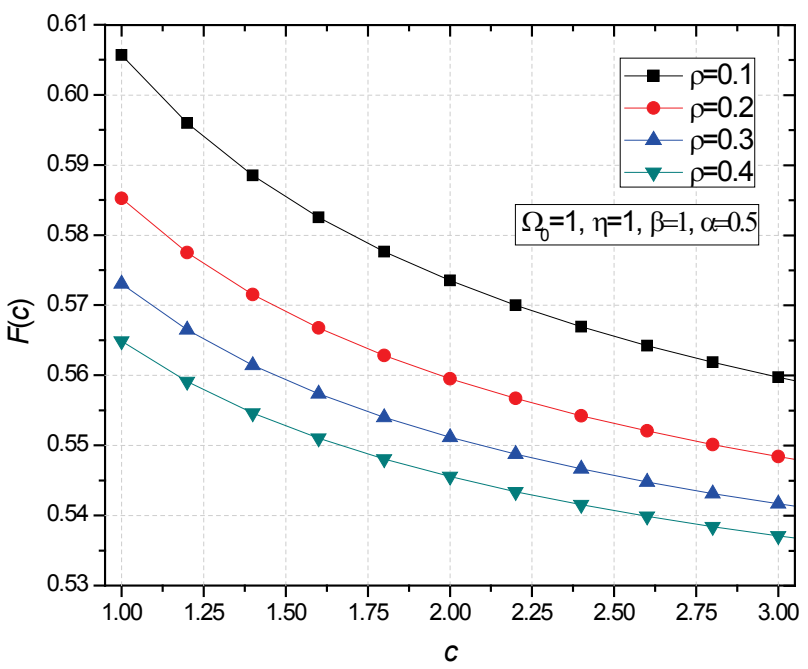

Figure 3 Cumulative distribution function versus Gamma long term fading severity parameter $c$

\section{CONCLUSION}

The MAD technique with the MAD SC receiver and two MID SC receivers are operating on the shaded multilayer pale channels in the presence of the co-channel interference subjected to short-term fading. Received derived signal and co-channel interference experience Weibull multipath fading and correlated Gamma long term fading. MAD SC receiver reduces Gamma long term fading effects and MID SC receivers reduce Weibull short term effects fading on system performance. MAD SC receiver selects MID SC receiver with higher signal envelope average power to provide services to user and MID selects and outputs branch with the higher ratio of signal envelope and interference envelope resulting in system performance improvement. Closed form expression for cumulative distribution function for signals at outputs MID SC receivers is calculated. By using these formulas, cumulative distribution function of MAD SC receiver output signal envelope is efficiently calculated. Outage probability of MAD system can be evaluated from cumulative distribution. The influence of Gamma long term fading severity parameter, Gamma long term fading correlation coefficient and Weibull short term fading nonlinearity parameter on outage probability is analysed and discussed. The system performance is better for lower values of outage probability. Outage probability decreases when Gamma shadowing severity parameter increases. Outage probability has higher values for lower values of Weibull nonlinearity parameter. The influence of Weibull parameter on outage probability is higher for lower values of Gamma fading severity parameter. When correlation coefficient goes to one, the least signal occurs simultaneously on both MID SC receivers. In this case, MAD system becomes MID system.

\section{REFERENCES}

[1] Stuber, G. L. (2003). Mobile communication, $2^{\text {nd }}$ ed. Kluwer Academic Publisher, Dordrecht.

[2] Simon, M. K. \& Alouini, M. S. (2000). Digital Communication over Fading Channels. John Wiley \& Sons, USA. https://doi.org/10.1002/0471200697

[3] Panic, S., Stefanovic, M., Anastasov, J., \& Spalevic, P. (2013). Fading and Interference Mitigation in Wireless Communications. CRC Press, USA. https://doi.org/10.1201/b16275

[4] Mukherjee, S. \& Avidor, D. (2003). Effect of Microdiversity and Correlated Macrodiversity on Outages in a Cellular System. IEEE Transactions on Wireless Technology, 2(1), 50-59. https://doi.org/10.1109/TWC.2002.806363

[5] Shankar, P. M. (2008). Analysis of microdiversity and dual channel macrodiversity in shadowed fading channels using a compound fading model. International Journal of Electronics and Communications (AEUE), 62(6), 445-449. https://doi.org/10.1016/j.aeue.2007.06.008

[6] Yacoub, M. D. (2007). The $\eta-\mu$ distribution and the $\kappa-\mu$ distribution. IEEE Antennas and Propagation Magazine, 49(1), 68-81. https://doi.org/10.1109/MAP.2007.370983

[7] Malmgren, G. (1997). On the performance of single frequency networks in correlated shadow fading. IEEE Transactions on Broadcasting, 43(2), 155-165. https://doi.org/10.1109/11.598364

[8] Goldsmith, A. (2005). Wireless communications. Cambridge University press, Cambridge, UK. https://doi.org/10.1017/CBO9780511841224

[9] Sekulovic, N., Stefanovic, M., Milovic, D., \& Stanojcic, S. (2014). Second-Order Statistics of System with N-branch Microdiversity and L-branch Macrodiversity Operating over Gamma Shadowed Nakagami-m Fading Channels. International Journal of Communication Systems, 27(2), 390-400. https://doi.org/10.1002/dac.2369

[10] Stefanovic, D., Panic, S., \& Spalevic, P. (2011). Secondorder statistics of SC macrodiversity system operating over Gamma shadowed Nakagami-m fading channels. $A E U$ International Journal of Electronics and Communications, 65(5), 413-418. https://doi.org/10.1016/j.aeue.2010.05.001

[11] Gradshteyn, I. S. \& Ryzhik, I. M. (2000). Table of Integrals, Series and Products. Academic Press, San Diego, USA. 


\section{Contact information:}

\section{Miloš PERIĆ,}

Faculty of Electronic Engineering, University of Niš

Aleksandra Medvedeva 14, 18000 Niš, Serbia

E-mail:milosperic@yahoo.com

\section{Branimir JAKŠIĆ,}

Faculty of Technical Sciences, University of Priština

Knjaza Miloša 7, 38220 Kosovska Mitrovica, Serbia

E-mail: branimir.jaksic@pr.ac.rs

\section{Danijela ALEKSIĆ,}

College of Applied Technical Sciences

Aleksandra Medvedeva 20, 18000 Niš, Serbia

E-mail: danijela.aleksicnikolic@gmail.com

\section{Dragan RANDJELOVIĆ,}

Academy of Criminalistic and Police Studies

Cara Dusana 196, 11080 Belgrade, Serbia

E-mail: dragan.randjelovic@kpa.edu.rs

\section{Mihajlo STEFANOVIĆ,}

Faculty of Electronic Engineering, University of Niš

Aleksandra Medvedeva 14, 18000 Niš, Serbia

E-mail: misa.profesor@gmail.com 